\title{
Optimal Measurements for Detection of Coronary Artery Disease by Exercise Electrocardiography
}

\author{
MAARTEN L. SimOONS \\ Thoraxcenter, Erasmus University, Rotterdam, The Netherlands
}

Received December 17,1976

\begin{abstract}
Exercise electrocardiograms were analysed by digital computer in order to select criteria for optimal separation of normal subjects and patients with coronary artery disease. The results of a training group of 86 ostensibly healthy men and 52 patients were verified in a test series of 43 patients and 43 controls. All subjects had a normal $Q R S$ complex and $S T-T$ segment at rest. ECG measurements included $S T$ amplitudes and slopes at fixed intervals after the end of $Q R S$, time-normalized $S T$ amplitudes, the negative $S T$ areas, and Chebyshev waveform vectors. Visual reading of the ECG according to the classical criterion of a $0.1 \mathrm{mV}$ horizontal $S T$ depression yielded a sensitivity of 50 and $51 \%$ and a specificity of 94 and $95 \%$ in the training group and the test group, respectively. Best results with a single $S T$ measurement were obtained by the $S T 60$ amplitude in lead $X$ which was adjusted for heart rate according to the gradual ECG changes during exercise in the normal subjects. The sensitivity was $81 \%$ and the specificity $93 \%$. In the test group these figures were 70 and $93 \%$. Combination of measurements in linear discriminant functions resulted in some further improvement of these figures.
\end{abstract}

\section{INTRODUCTION}

A horizontal depression of the $S T$ segment in an electrocardiogram recorded during exercise (XECG) in male patients with chest pain is a specific predictor of obstructive lesions in the coronary arteries, provided that no signs of other cardiac abnormalities are present (1-5). However, these studies also indicate that in onethird of the patients with abnormal coronary arteries the XECG remains within normal limits when the tracings are read by human observers. Part of this discrepancy between the XECG and selective coronary arteriography is due to problems in interpretation of the ECG from muscle noise, drift, and motion artifacts during exercise. Computer processing may enhance the signal quality by selective averaging. In addition, more accurate measurements can be taken and more sophisticated statistical methods may be used to separate normal and abnormal ECG responses during exercise with the aid of a computer system. Therefore several authors have attempted to improve the diagnostic value of the XECG by means of computer processing.

Horsten and Bruce (6) developed a system for analysis of a single bipolar lead which is currently in on-line operation. Mcasurcments are taken of the $S T$ amplitude at fixed intervals after the nadir of the $S$ wave. No methods have been built in this 
system to prevent incorporation of premature beats and normal beats into a single averaged complex. Also, no data have been presented thus far on the sensitivity and specificity of the criteria employed. Thus the clinical reliability of this system is not established. McHenry et al. (7) combined the amplitude and slope of the $S T$ segment in lead $V_{5}$ at a fixed interval after the end of the $Q R S$ complex. They reported, on a series of 166 patients, a sensitivity of $82 \%$ and a specificity of $95 \%$. Unfortunately the clinical usefulness of this method remains in doubt because no data were presented on the results of visual interpretation of the XFCGs in these patients. The area of the $S T$ segment below the baseline was proposed by Sheffield $e t$ al. $(8)$ as a measure for ischemia during exercise. A satisfactory separation was obtained in a first series of 41 normal subjects and 38 patients with typical angina pectoris. Blomqvist (9), in 1965, employed time-normalized $S T$ amplitudes from the Frank leads and linear discriminant function analysis to separate XECGs from small series of normal subjects and patients with CAD. No further data on this system have been reported since. An analogue computer was introduced by Dower et al. (10) to derive the polar coordinates of the heart vector after exercise. These authors proposed a combination of the spatial magnitude and orientation of ST vectors for interpretation of XECGs. This criterion has not yet been tested clinically. Normal values for Chebyshev waveform vectors which describe the $S T$ segment at rest and during exercise were computed by Rautaharju et al. (11). This method has also not vet been tested in patients with CAD. Part of the criteria described above $(7,8)$ were utilized by Ascoop et al. $(12,13)$ in a series of 87 patients who underwent selective cine-coronary arteriography and 15 asymptomatic normal subjects. These authors also derived some new criteria for classification of orthogonal and bipolar XECGs. Best separation of subjects with and without significant coronary artery obstructions was obtained with a combination of the $S T$ amplitude and the slope in bipolar lead $C M_{5}$. These results have recently been confirmed in an independent test series (14).

On the basis of these encouraging results a systematic analysis of the XECG in a series of ostensibly healthy subjects and patients with independently proven CAD and a normal ECG at rest was undertaken. Each of the measurements described by these authors $(6-13)$ was tested. Since in a previous study (15) in normal subjects gradual ECG changes were observed during exercise. an attempt was made to improve the diagnostic classification of the XECG by correction of the $S 7$ measurements for instantaneous heart rate. As will be shown, this method indeed enhanced the sensitivity of the interpretation of the records. Finally, linear disciminant function analysis was employed to select combinations of measurements which optimally separated the XECGs of normals and patients. The results of this multivariate analysis were compared with other criteria in an independent test series of normal subjects and patients with coronary artery disease.

\section{Patients, Controls, and Methods}

Exercise electrocardiograms were analysed from 95 male patients with documented coronary artery disease. One hundred and twenty-nine ostensibly healthy men 
were used as a normal reference group. Forty-one patients had $70 \%$ or greater narrowings in the coronary arteries demonstrated by selective cine-coronary arteriography. Forty-one other patients were selected because they developed typical angina pectoris during the exercise test which disappeared within $5 \mathrm{~min}$ in the recovery period. The remaining 30 patients were tested in the first year after a myocardial infarction. All patients had a normal $Q R S$ complex and $S T$ segment at rest according to the Minnesota code. The normal subjects were selected from a population survey in Rotterdam because they had a low risk for development of coronary artery disease (15). All subjects underwent a graded exercise test on a bicycle ergometer with stepwise workload increments of $10 \mathrm{~W} / \mathrm{min}$ until near exhaustion, unless other symptoms appeared which required termination of the test
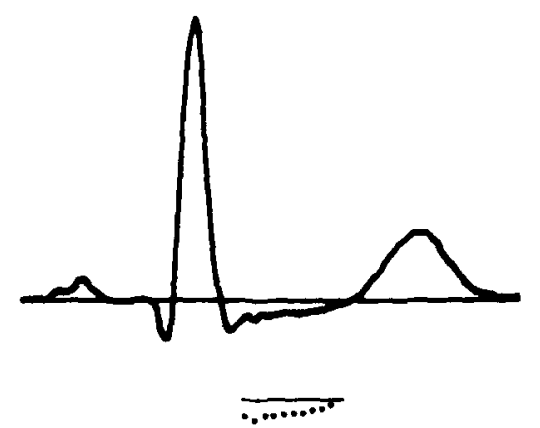

FIG. 1. ECG, lead $X$. The dots represent the $S T$ amplitudes at $10-\mathrm{msec}$ intervals after the end of QRS.

at an earlier stage (15). The Frank lead ECG was recorded on magnetic tape and analysed by digital computer with the method described in detail elsewhere (16). ECG measurements were taken every second or third minute during exercise and in the first $4 \mathrm{~min}$ of the recovery period. These included the $P, Q, R$, and $S$ amplitudes in all three leads, $S T$ amplitudes and slopes at 10 - $\mathrm{msec}$ intervals from 10 to $90 \mathrm{msec}$ after the end of $Q R S(7,12,13), 1 / 8$ time-normalized $S T$ amplitudes and slopes (9), the negative $S T$ area (8), and the first six coefficients of Chebyshev polynomials fitted to the $S T$ interval (II). Details of these measurements have been presented in Figs. 1 to 4 . In addition, the polar coordinates of the measurements were analysed.

The goal of the present study was to develop and to test algorithms for classification of XECGs into two groups: "normal" or "exercise-induced ischemia." Following the arguments of Cornfield (17) linear discriminant functions can be derived to separate these two categories provided that they are mutually exclusive and exhaustive, which means that no other categories exist. It should also be known, without error, to which category each individual truly belongs. The selection criteria outlined above provided indeed a reference population and a group of patients with coronary artery disease which has been documented without prior knowledge of the XECG. These two groups may be considered mutually exclusive and exhaustive. Thus linear discriminant functions could be derived for classification of subjects with 
a normal resting ECG into "normal" and "exercise-induced ischemia" on the basis of the XECG alone. Furthermore, computation of linear discriminant functions assumes a multivariate normal distribution of independent data and equal covariance matrices in the two groups (17). This is not entirely true for some of the parameters

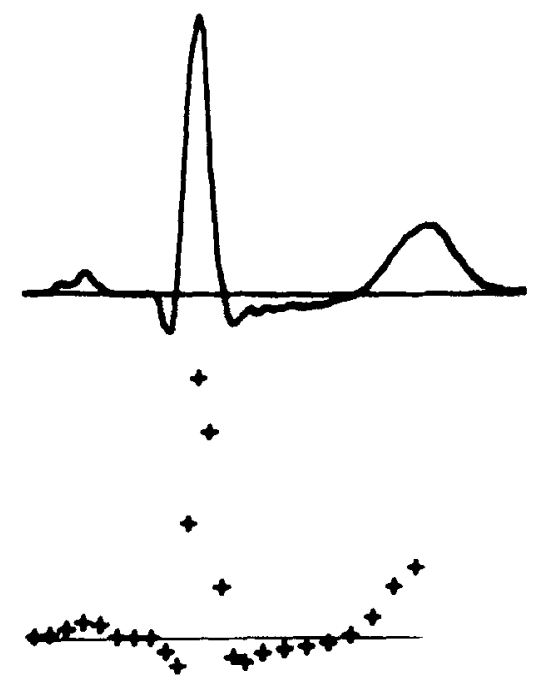

FIG. 2. Time-normalized amplitudes in the $P Q$ interval, the $Q R S$ complex, and the $S T$ interval. The points represent the mean amplitudes at eight equal parts of these intervals. The $3 / 8$ point in the $P Q$ interval corresponds to the maximum spatial magnitude of the $P$ wave. The $8 / 8 S T$ amplitude corresponds to the maximum spatial magnitude of the $T$ wave.

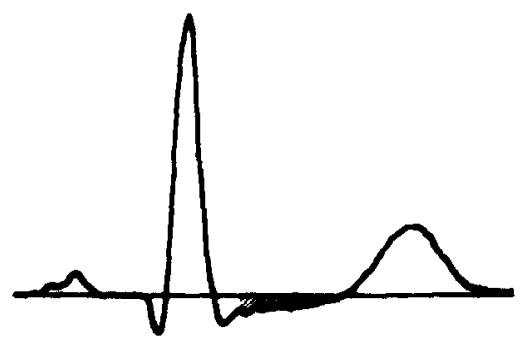

FIG. 3. The hatched area represents the integral of the negative part of the $S T$ interval.

proposed above. However, Cornfield (I7) has shown that the results can still be useful, even when multivariate normality is not present. In that case, it is necessary to validate the results of the analysis in an independent test series. Accordingly, the data were divided in a training set which consisted of 86 normal subjects and 52 patients, and an independent test set of 43 normals and 43 patients. 
The linear discriminant function analysis was performed with a standard program at the University Computer Center in Utrecht utilizing a CDC 7600 system. This program generated discriminant functions with all possible combinations of at most three parameters. This limitation was chosen because the series were relatively small. The results of different algorithms for separation of normals and patients with coronary artery disease were expressed in terms of the sensitivity (fraction of
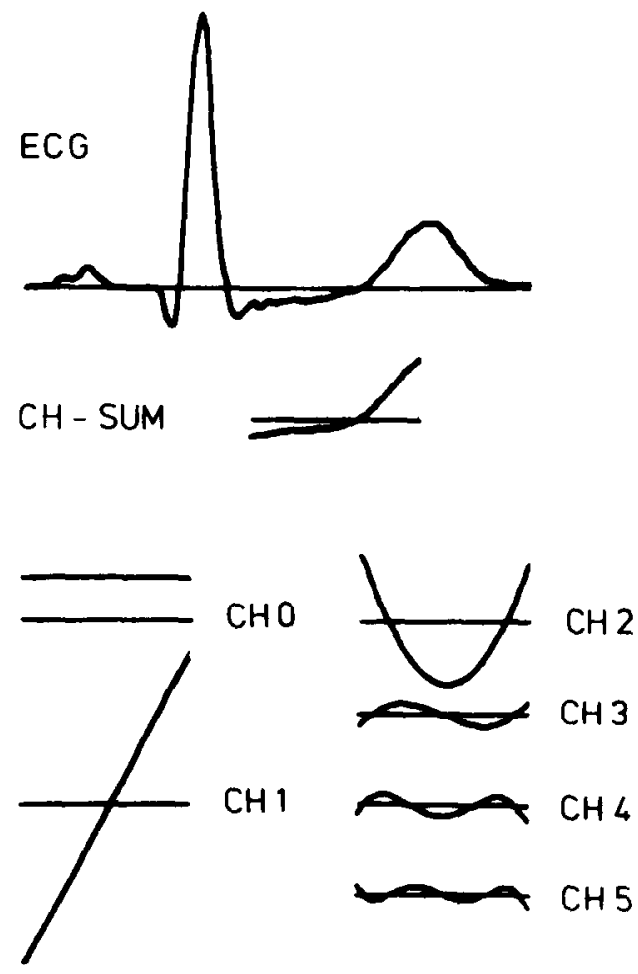

FIG. 4. The $S T$ segment can be described by six Chebyshev polynomials. In the lower part of the figure the individual polynomials have been plotted $(\mathrm{CHO}, \mathrm{CH} 1-\mathrm{CH})$ with the correct relative strength. $\mathrm{CH}$-SUM is the $\mathrm{ST}$ segment which was obtained by combination of the six polynomials. Note the excellent reproduction of the $S T$ segment of the original ECG on top.

abnormal findings in the patients) and the specificity (fraction of normal findings in the control group). These two were then combined in a single Index of Merit, which is the sum of sensitivity and specificity minus one (12).

\section{RESULTS}

In Fig. 5 the distribution of the highest heart rate during exercise in the four groups is presented. The peak heart rates in the patients were lower than in the 

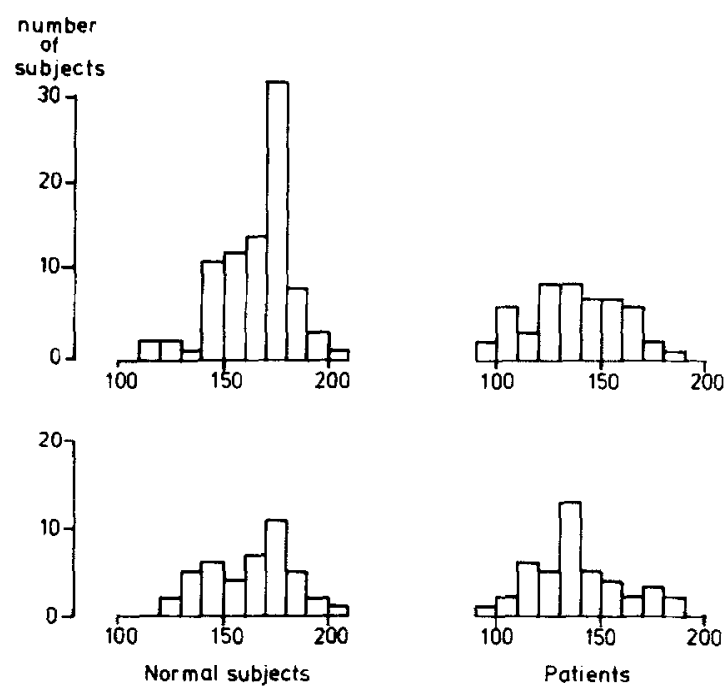

FIG. 5. Histograms of the highest heart rates during exercise in the normal subjects (left) and in the patients (right) from the training group (top) and the test group (bottom). Note that the highest heart rates in the patients were considerably lower than in the normal subjects. The heart rates in the training group and test group were similar.

normal subjects, but the heart rates in the training and test group were similar. In most normal subjects the peak heart rates were close to the age-predicted maximum; in some however, the test was terminated at an earlier stage.

\section{ECG at Rest}

The $P$ waves, $Q R S$ complexes, and $S T$ segments in the patients at rest were similar to those in the normal subjects. Multivariate analysis was applied to the ECG measurements at rest. The best results are summarized in Table I. Single

\section{TABLE I}

Multivariate analysis of eCG Measurements AT REST FROM 86 NORMAL SUBJECTS AND 52 Patients with Coronary artery Disease and a Normal Resting ECG ${ }^{a}$

\begin{tabular}{lccc}
\hline Measurements & Spec. & Sens. & Index \\
\hdashline$S T 90_{x}$ & 0.63 & 0.62 & 0.24 \\
$S T 50_{x} . S T 80_{y}$ & 0.65 & 0.65 & 0.31 \\
$S T 50_{x}, S T 70_{z}, S T 80_{1}$ & 0.67 & 0.67 & 0.35 \\
\hline
\end{tabular}

"ST10 $\ldots S T 90=$ amplitudes at $10 \ldots 90 \mathrm{msec}$ after the end of $Q R S$. The subscripts $x, y, z$ denote the three leads of the Frank system. Spec. $=$ specificity; Sens. $=$ sensitivity; Index $=$ Index of Merit. 
measurements from the $P Q, Q R S$, or $S T$ segments in the patients and controls overlapped almost completely. Even combination of three measurements in a linear discriminant function yielded a poor separation of the two groups. The Index of Merit was 0.35 , sensitivity was $67 \%$, and specificity was $67 \%$. This indicates that the resting ECGs in the patients were indeed almost identical to those in the controls.

\section{ECG during Exercise}

The results of the discriminant function analysis of measurements at maximum heart rate have been summarized in Table II. The negative area of the $S T$ segment in lead $X\left(S T A_{x}\right)$ yielded an Index of Merit of 0.52. Combination with measurements from the $Q R S$ complex $\left(Q_{x}, Q_{y}\right)$ and $T$ wave $\left(T A_{x}\right)$ increased the index to 0.65 . The best discriminating Chebyshev coefficients were the mean $S T$ amplitude $\left(\mathrm{CHO}_{x}\right)$ and the $S T$ gradient $\left(\mathrm{CH}_{x}\right)$. The highest Index of Merit obtained with three parameters was only 0.61 . The indices obtained with the time-normalized $S T$ amplitudes were in

TABLE II

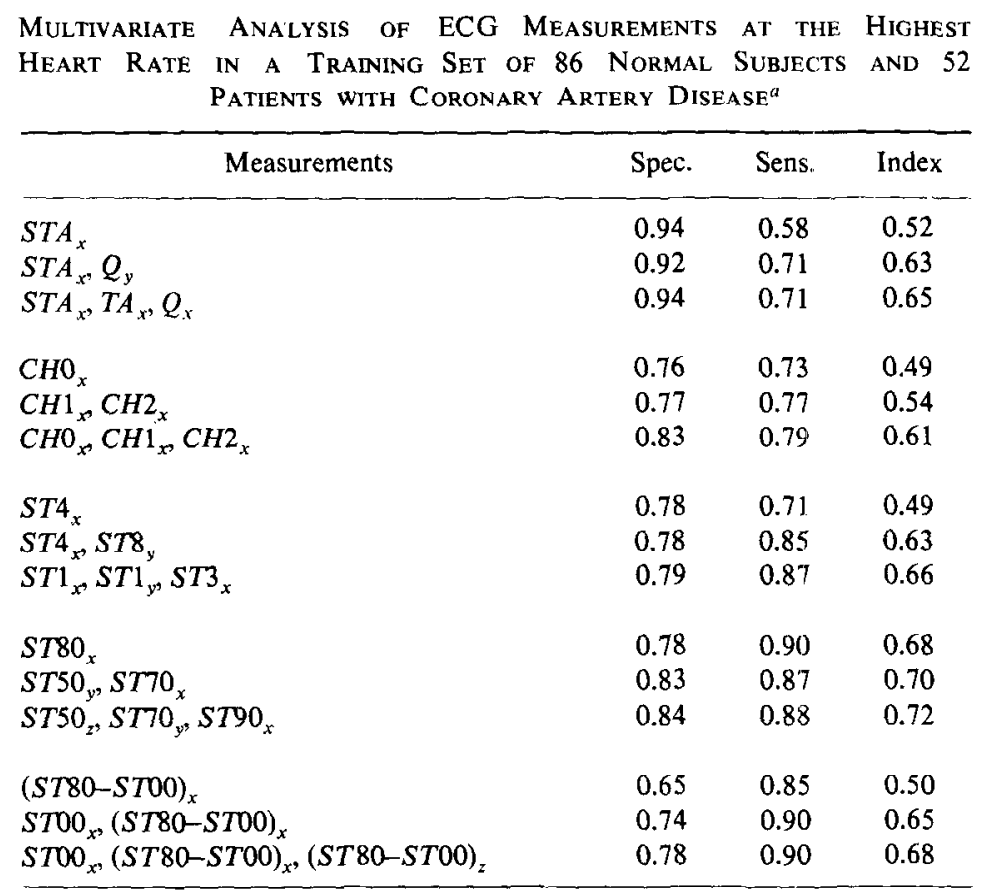

${ }^{a} S T 1 \cdots S T 8=$ time normalized $S T$ amplitudes at $1 / 8 \cdots 8 / 8$ from the end of $Q R S$ until the maximum spatial magnitude of the $T$ wave; $\mathrm{CHO} \cdots \mathrm{CH} 6=$ Chebyshev waveform vectors. (ST80-ST00) indicates the slope of the $S T$ segment from $S T 00$ until $S T 80$. STA = area of negative part of $S T$ segment; $T A=$ area of positive part of $T$ wave; $Q=$ amplitude of $Q$ wave. See legend Table I. 
the same range. It should be noted that not only amplitudes from the midportion of the $S T$ segment were found to be of discriminative value, but also amplitudes near the maximum magnitude of the $T$ wave $\left(S T 8_{y}\right)$ and amplitudes close to the $S T$ junction $\left(S T 1_{x}, S T 1_{y}\right)$. The best single measurement for separation of patients and normals was $S T 80_{x}$, Index of Merit 0.68 . Combination of two $S T$ amplitudes at a fixed interval after the end of $Q R S$ yielded an Index of Merit of 0.70 . Also little further improvement was obtained by combination of three $S T$ amplitudes. Combination of $S T$ amplitudes and $S T$ slopes did not give better results than the $S T$ amplitudes alone. In addition, the separation of normal subjects and patients could not be enhanced by combination of $S T$ areas, $S T$ amplitudes, and $S T$ slopes in one discriminant function.

Dower et al. (10) proposed the use of polar coordinates instead of Cartesian coordinates for classification of XECGs. In order to test the value of this approach, the $S T$ vectors were expressed as the spatial magnitude $(M)$, the frontal plane angle $(F)$, and the angle in the transverse, horizontal, plane $(T)$. Both angles were measured relative to the $X$ axis. Since angular measurements are not normally distributed, these were transformed prior to multivariate analysis, as described by Cornfield et al. (18). Representative results in Table III show that the directions of the $S T$ vectors were more discriminative than the vector magnitudes. The $S T$ vectors at a fixed interval after $Q R S$ again yielded better separation of patients and controls than the time-normalized $S T$ vectors and the Chebyshev waveform vectors. The best single parameter was the direction in the frontal plane of $S T 80$, with an Index of Merit 0.66. The highest index which could be obtained with three parameters was 0.71 . These results are almost identical to those obtained with Cartesian coordinates in Table II. This indicates that analysis of the polarcardiogram during exercise in patients with a normal ECG at rest does not improve the diagnostic value of the XECG.

TABLE III

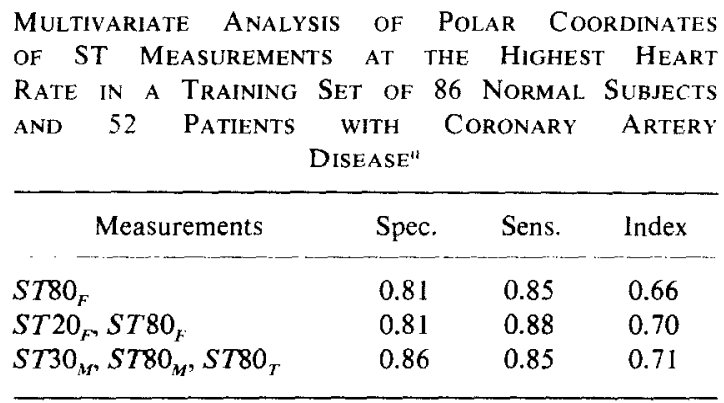

${ }^{a}$ Note that the Indices of Merit for the polar coordinates were almost identical to those for the $X, Y$, and $Z$ components in Table II. $M=$ magnitude of a given vector; $F=$ frontal plane angle; $T=$ transverse plane angle. (See also legend to Table I.) 


\section{ECG Changes during Exercise}

In a given patient, the changes of the ECG during exercise can be described as the difference between the measurements at rest and those at the highest work load. This approach reflects the influence of exercise on the ECG independent of the variation in the ECG measurements at rest.

It was attempted to improve the diagnostic classification of the XECG by analysis of these changes instead of the measurements at the highest workload. In Table IV the results of discriminant function analysis of the ECG changes are summarized. The Indices of Merit were close to those at maximum heart rate (Table II). Combination of $S T$ slopes and $S T$ amplitudes and transformation to polar coordinates did not improve the separation of patients and normal subjects.

TABLE IV

\begin{tabular}{|c|c|c|c|}
\hline \multicolumn{4}{|c|}{ 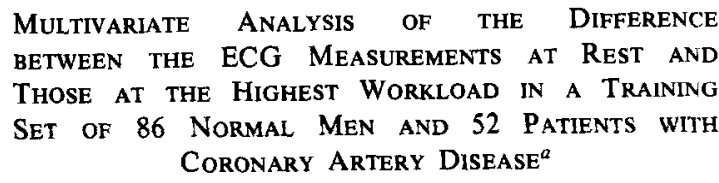 } \\
\hline Measurements & Spec. & Sens. & Index \\
\hline $\begin{array}{l}\Delta S T 80_{x} \\
\Delta S T 80_{x}, \Delta S T 90_{y} \\
\Delta S T 80_{x}, \Delta S T 80_{z}, \Delta S T 90_{y}\end{array}$ & $\begin{array}{l}0.84 \\
0.88 \\
0.87\end{array}$ & $\begin{array}{l}0.75 \\
0.77 \\
0.88\end{array}$ & $\begin{array}{l}0.59 \\
0.65 \\
0.75\end{array}$ \\
\hline
\end{tabular}

${ }^{a}$ For legend see Tables I and II.

\section{Correction for Instantaneous Heart Rate}

In the upper panel of Fig. 6 the area of the ST segment below the baseline (STA) in lead $X$ has been plotted against heart rate. In the reference population, these points fill a triangular field with the larger, more negative $S T A_{x}$ occurring at higher heart rates. Two straight lines could be computed which formed the boundaries of this field. For this purpose the upper and lower fifth percentiles were computed for all measurements at heart rates within the range $60-69,70-79$ beats per min, etc. Linear regression lines were computed through the upper and lower fifth percentiles. These lines have been drawn in the plot (I and II). Many measurements in the patients were found below line II, while most measurements from the controls fell above this line. When the measurements below line II in Fig. 6 were labeled abnormal, the sensitivity of $S T A_{x}$ for prediction of coronary artery disease was $73 \%$. This was considerably better than the sensitivity obtained with the same absolute threshold value for all heart rates as shown in Table V. ST amplitudes in lead $X$ yielded even higher sensitivities when heart rate dependent normal ranges 


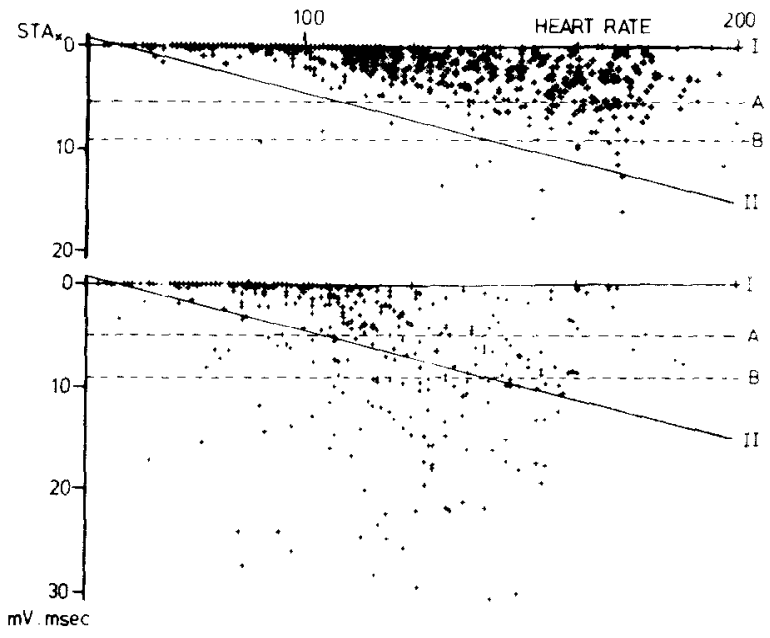

FIG. 6. Plot of the negative $S T$ areas at rest and during exercise in 86 normal subjects (top) and $\$ 2$ patients with coronary artery disease (bottom). Lines I and II represent the boundaries of the area in which the measurements from the normal subjects were found. The dotted lines $A$ and $B$ correspond with a $S T A_{x}$ of 5 and $9 \mathrm{mV} \times$ msec. See text for further explanation.

were used: for $S T 4_{x}$ and $S T 60_{x}$ the sensitivities were 83 and $81 \%$ when the specificities were kept at $93 \%$.

Linear discriminant function analysis hardly improved the separation of patients and normals by this type of measurements as demonstrated in Table VI.

TABLE V

Comparison of absolute and Heart Rate-Dependeni Crileria hok INTERPRETATION OF XECGS IN A TRAINING SET OF 86 NORMAL SUBJECTS and 52 Patients With Coronary Artery Disease ${ }^{a t}$

\begin{tabular}{|c|c|c|c|c|c|c|}
\hline \multirow[b]{2}{*}{ Measurements } & \multicolumn{3}{|c|}{ Absolute threshold } & \multicolumn{3}{|c|}{ Heart rate dependent } \\
\hline & Spec. & Sens. & Index & Spec. & Sens. & Index \\
\hline$S T A_{x}$ & 0.94 & 0.58 & 0.52 & 0.93 & 0.73 & 0.66 \\
\hline$S T 4_{x}^{x}$ & 0.92 & 0.71 & 0.63 & 0.93 & 0.83 & 0.76 \\
\hline$S T 60_{x}$ & 0.95 & 0.63 & 0.59 & 0.93 & 0.81 & 0.74 \\
\hline
\end{tabular}

${ }^{4}$ For legend see Tables I and II.

\section{Trend Analysis}

In normal subjects the variance of some $S T$ amplitudes was significantly reduced by prediction from instantaneous heart rate with linear regression equations (15). Also linear regression lines can be computed to express the relation between $S T$ 


\section{TABLE VI}

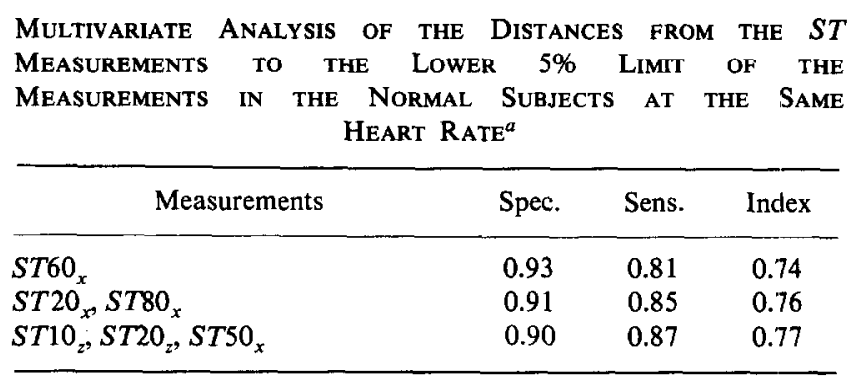

${ }^{a}$ For legend see Table I and Fig. 6.

measurements and heart rate in one individual. For example $S T 70_{x}$ can be expressed as $S T 70_{x}=A+B \times \mathrm{HR}$. The slope $B$ of this equation is the rate of change of $S T 70_{x}$ during the stages of the exercise test. This measurement, together with the intercept $A$ which is the hypothetical value of $S T 70_{x}$ at zero heart rate and with the standard error of the estimate, was chosen as a representative of the trend of $S T 70_{x}$. It could be expected that these figures would improve the classification of the XECG when compared with measurements at the highest work load only. Therefore the intercepts, slopes, and standard errors of the estimate of these regression lines were computed for all individuals and entered in the discriminant analysis. Results are presented in Table VII. The analysis of the trend of the negative $S T$ areas resulted in a lower sensitivity than analysis of the values at maximum heart rate. A marked improvement was obtained with trend analysis of time-normalized $S T$ amplitudes, while the Indices of Merit of the amplitudes at fixed intervals after $Q R S$ were similar

TABLE VII

Multivariate ANALYsis OF INTERCEPts (A) AND Slopes (B) of Linear Regression Lines Relating ST Measurements AND Instantaneous Heart Rate in Individual SubJects of the Training Set of 86 Normal SubJects and 52 Patients With Coronary Artery Disease ${ }^{a}$

\begin{tabular}{lccc}
\hline \multicolumn{1}{c}{ Measurements } & Spec. & Sens. & Index \\
\hline$B-S T 4_{x}$ & 0.92 & 0.69 & 0.61 \\
$A-S T 4_{x}, B-S T 4_{x}$ & 0.96 & 0.71 & 0.67 \\
$A-S T 4_{x}, B-S T 4_{x}, A-S T 4_{z}$ & 0.96 & 0.79 & 0.75 \\
$B-S T 70_{x}$ & & & \\
$B-S T 80_{x}, B-S T 80_{z}$ & 0.93 & 0.75 & 0.68 \\
$A-S T 80_{x}, B-S T 80_{x}, B-S T 80_{z}$ & 0.88 & 0.83 & 0.71 \\
\hline
\end{tabular}

\footnotetext{
${ }^{a}$ For legend, see Table I.
} 
to those in Table II. However, the separation of patients and normals obtained in this manner was not better than the separation obtained with amplitudes adjusted for instantaneous heart rate (Table V).

Comparison between Different Criteria for Two-Group Classification of XECGs in the Training Set and in the Independent Test Series

A number of selected criteria for interpretation of XECGs were compared in the training group of 86 normal subjects and 52 patients and in an independent test group of 43 normal men and 43 patients (Table VIII).

A horizontal $S T$ depression of $0.1 \mathrm{mV}$ or more was observed in $50 \%$ of the patients in the training group and in $51 \%$ of those in the test group. The specificity of visual XECG interpretation by an experienced electrocardiographer was 94 and $95 \%$ in the two groups. In spite of the excellent agreement between the results of visual XECG interpretation, the sensitivity of most computer measurements in the test group was lower than in the training group. In particular a poor reproducibility was observed in the discriminant functions which combined two or more measurements. There were two exceptions to this finding: the combination of the heart rate adjusted ST20 and ST80 amplitudes in lead $X$ and the trend of ST4 in the same lead. The former yielded a sensitivity of $84 \%$ and specificity of $88 \%$ in the test group, which is a considerably better separation of patients and normals than the one obtained by visual ECG reading.

\section{ST Measurements}

\section{Discussion}

A whole range of measurements have been proposed previously $(6-13)$ for interpretation of electrocardingrams recorded during exercise. In the present study, a systematic comparison of these measurements was made with the aid of multivariate statistical methods in order to evaluate their usefulness for detection of coronary artery disease.

$S T$ amplitudes at a fixed interval after the end of $Q R S(6,7)$ can easily be obtained both by computer and by hand. However, a theoretical disadvantage of these measurements is that the shortening of the $Q T$ interval at higher heart rates is not taken into account. Therefore, time-normalized $S T$ amplitudes have been proposed (9. 18). Computation of these measurements requires localization of a reference point in the $T$ wave during exercise. The end of the $T$ wave cannot be used for this purpose since it is usually buried under the next $P$ wave at heart rates higher than 120 beats per min. In the present study the maximum spatial magnitude of the $T$ wave was chosen as a reference point. In a few cases this point could also not be defined. especially in those records where the $S T$ loop was deformed into a "horseshoe" shape during myocardial ischemia (19).

An accurate mathematical description of the $S T$ segment is provided by the Chebyshev waveform vectors proposed by Rautaharju et al. (11). Only four to six 


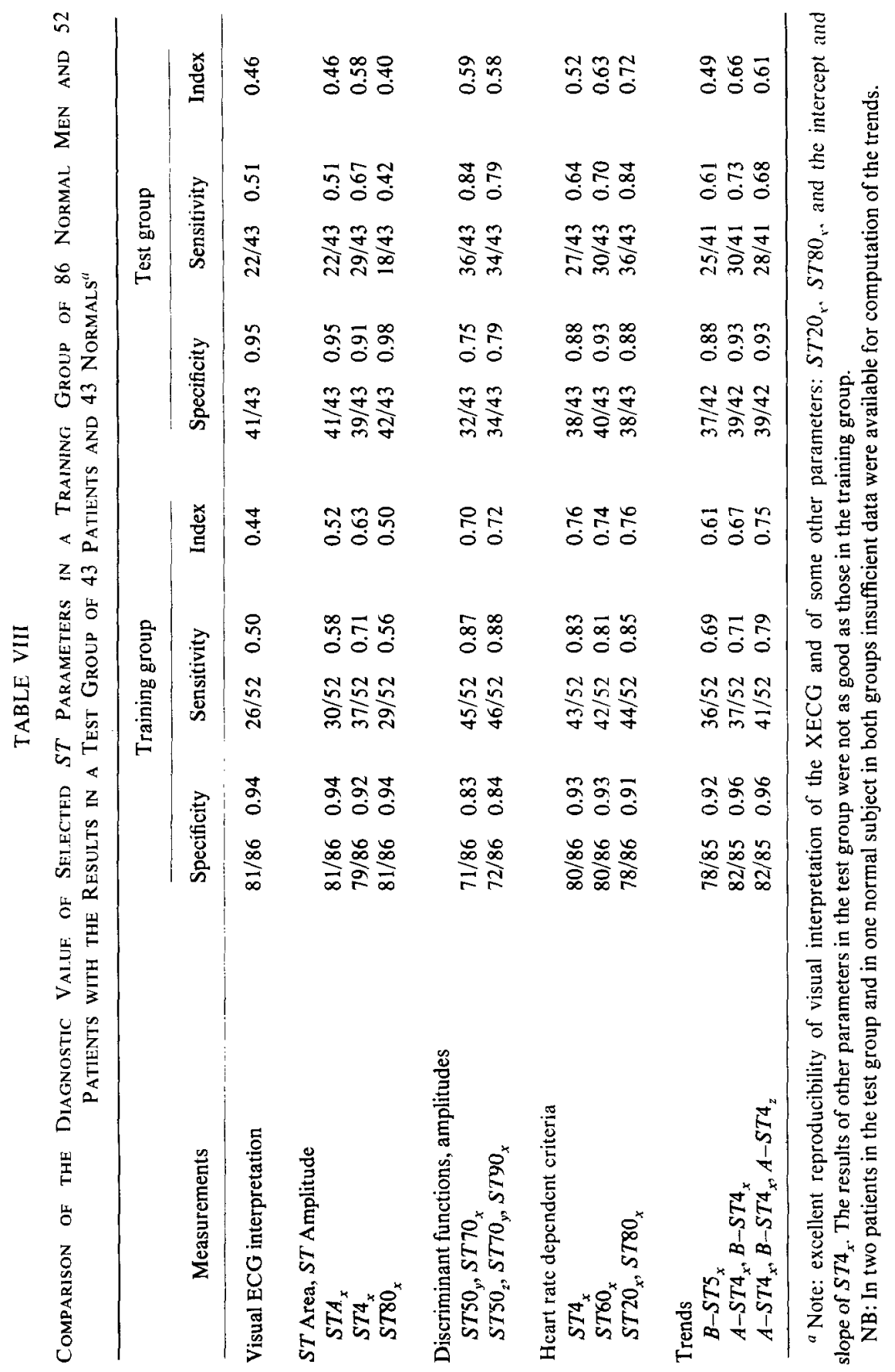


Chebyshev coefficients are required to reproduce the shape of the $S T$ segment as demonstrated in Fig. 4.

Systematic analysis of these measurements in a training group of 86 normal subjects and 52 patients with CAD led to the conclusion that the level of the $S T$ segment below the baseline is the best discriminating factor. $S T$ amplitudes at a fixed interval after the end of $Q R S$ yielded better results than time-normalized $S T$ amplitudes, the $S T$ areas, or the Chebyshev waveform vectors (Table II). The addition of the $S T$ slopes did not improve the diagnostic classification nor did transformation to polar coordinates (Table III) or measurement of the changes during exercise (Table IV).

\section{Correction for Instantaneous Heart Rates}

In a previous study (15), we observed that many ECG measurements gradually change during exercise in normal subjects. Therefore it was expected that adjustment of ECG measurements for instantaneous heart rate would improve the separation of XECGs from normal subjects and patients with coronary artery disease. This was confirmed both in the training group (Table $\mathrm{V}$ ) and in the test group (Table VIII). This indicates that a certain amount of $S T$ depression in lead $X$ may be found in normal subjects at high heart rates, while the same depression is a sign of myocardial ischemia. when it occurs at a low heart rate. Therefore, it is concluded that the adjustment of $S T$ measurements for heart rate as described in this study considerably improves the diagnostic value of exercise electrocardiography.

\section{Multivariate Analysis}

Introduction of multivariate statistical methods has improved the diagnostic classification of the majority of resting ECGs (18). It has also been applied to obtain a clinical index for symptomatic coronary artery disease $(20)$. This method was used in the present study in order to predict the presence or absence of CAD by linear combination of various $S T$ measurements. The discriminant functions developed in the training group of patients and controls were applied to an independent test group in order to detect too optimistic results which may be found in a relatively small data base.

The results presented in Tables II to IV demonstrate indeed some improvement of the separation of patients and normals when multiple measurements were combined. For instance, the highest Index of Merit for single $S T$ measurements in Table II was 0.68 while an Index of 0.72 was obtained when three amplitudes from all three leads were taken into account. However, the improvement of the diagnostic classification by linear discriminant function analysis in the training group was less than expected. It appeared that the measurements which discriminated patients from normals were predominantly those from lead $X$. The addition of measurements from the other two orthogonal leads was usually of minor importance. This is in agreement with the data published by Ascoop (13).

The separation of normal subjects and patients was not as good in the test group as it was in the training group (Table VIII). This may be due in part to deviations 
from the underlying assumptions of the multivariate statistical theory. Furthermore, the lower Indices of Merit in the test group may be related to the small sample size and to the differences in patient selection. The majority of the patients in the training group, 32 out of 52 , were selected on basis of abnormal findings in the coronary arteriogram, while the test group consisted mainly of patients with angina pectoris during the exercise test, 30 out of 43 . This indicates the need for further study on larger populations. Nevertheless, an excellent reproducibility in the test group was found for the linear combination of two heart rate corrected $S T$ amplitudes from lead $X$ at the highest work load (Table VIII).

\section{Comparison with Other Studies}

The sensitivity of visual interpretation of the XECG in this study was $50 \%$. This is lower than in most studies reported by other authors $(1,2,4,5)$. Differences in patient selection are the most likely explanation for this finding. In the present study all patients with an abnormal $Q R S$ complex or abnormal $S T-T$ segment at rest were excluded, because an abnormal ECG at rest is already strong evidence for myocardial disease. Slight repolarization disturbances or intraventricular conduction abnormalities were not excluded in two studies in which a higher sensitivity of the XECG was reported $(1,5)$. Two other investigations even included patients with abnormal $Q R S$ complexes and $S T-T$ segments at rest $(2,4)$. In one other report the patient selection was limited to those without repolarization disturbances at rest (3). The results of visual interpretation in this series were very similar to ours: sensitivity $52 \%$, specificity $94 \%$, and Index of Merit 0.46 . Application of criteria for computerassisted interpretation of the XECG proposed by Ascoop (13) to the present material resulted in lower specificities and higher sensitivities than those reported by this author. For example, a critical value of $5 \mathrm{mV} \times \mathrm{msec}$ for the negative $S T$ area in lead $X$ resulted in a sensitivity of $44 \%$ and a specificity of $90 \%$ in the original report (13), while in the present study the sensitivity was $82 \%$ but the specificity only $76 \%$. This discrepancy can be explained with the aid of Fig. 6. The horizontal line $A$ represents a negative $S T$ area of $5 \mathrm{mV} \times \mathrm{msec}$. Ascoop et al. (13) frequently terminated the test at $90 \%$ of the age-predicted maximum heart rate, or even earlier. Thus these subjects did not reach the heart rates at which the larger, more negative, $S T$ areas were found. In the present study, however, most subjects were encouraged to exercise until exhaustion and in 21 out of 86 normal men from the training group (24\%), the $S T$ area exceeded $5 \mathrm{mV} \times$ msec. This observation again stresses the need for heart rate dependent criteria for interpretation of XECGs. The higher sensitivity in the present series is caused in part by the same difference in exercise protocol. Furthermore, it may depend on the patient selection, because in Ascoop's series most patients had a normal $S T$ response in a previous exercise test. Finally, part of the differences between these two investigations may be explained by the different methods for waveform analysis, since the negative $S T$ area is much influenced by the definition of both baseline and $Q R S$ offset. 
Two investigators $(7,13)$ observed an improvement of the classification of XECGs when the $S T$ amplitude was combined with the slope of the $S T$ segment. The criteria employed by these authors however, differ considerably (13). In the present study the $S T$ slopes were not superior to $S T$ amplitudes and a combination of both did not enhance the Index of Merit obtained by a combination of amplitudes alone (Table II). This is not as conflicting as it may seem, since a combination of the $S T$ amplitude and slope as computed by McHenry et al. (7) and Ascoop (13) is mathematically the same as a linear discriminant function with two $S T$ amplitudes.

\section{CONCLUSIONS}

Computer analysis of the XECG in the present study yielded higher sensitivity than visual ECG reading, both in the training group and in the independent test series. Optimal separation between patients with coronary artery disease and a normal ECG at rest and normal subjects was obtained when the $S T$ amplitudes were adjusted for the ECG changes during exercise in normal individuals. This adjustment was based solely on the relation between the ECG measurements and heart rate in the normal reference population. The criteria are therefore largely independent from the manner in which the patients with coronary artery disease were selected in the present study. A good reproducibility in the test group was obtained with the heart rate adjusted $S T 20$ and $S T 80$ amplitudes in lead $X$. These observations strongly favor the introduction of this type of measurement for routine interpretation of XECGs in subjects with a normal ECG rest. Some improvement of the diagnostic classification was obtained with multivariate analysis of the data at maximum heart rate in the training group. The results of this procedure in the test series were less satisfactory with two exceptions. Therefore further evaluation in a larger population is necessary before discriminant functions can be applied clinically. These studies are currently underway at our laboratory.

The best separation of patients and normal subjects was obtained with measurements from lead $X$ only. This suggests that a single, bipolar lead like $C B_{5}$ or $C M_{5}$ may be used instead of the more complex Frank system in those patients who have a normal ECG at rest. This is in accordance with other studies $(6,12,13)$ which compared the Frank leads with bipolar leads during exercise.

Heart rate dependent normal limits for the $S T$ amplitudes in bipolar leads can be established experimentally, or they can be computed by linear transformation of the data from the present series (21). In theory the heart rate adjusted $S T 60$ amplitude in lead $X$ or a bipolar lead can be used for visual interpretation of XECGs, provided that the quality of the tracings is good and the measurements are taken from a series of consecutive beats, which should be recorded with large amplification and on an expanded time scale. However, experience from our extensive studies indicates that the improved signal quality, the objective quality control, and the enhanced measurement accuracy strongly favor the addition of a small computer to the equipment in the exercise laboratory. 


\section{REFERENCES}

1. Ascoop, C. A.. Simoons. M. L., Egmond. W. G., And BruschKe. A. V. G. Exercise test, history, and serum lipid levels in patients with chest pain and normal electrocardiogram at rest; a comparison to findings in coronary arteriography. Amer. Heart J. 82, 609 (1971).

2. MARTIN, C. M. AND MCCONAHAY, D. R. Maximal treadmill exercise electrocardiography; correlations with coronary arteriography and cardiac hemodynamics. Circulation 46, 956 (1972).

3. Ascoop, C. A., Distelbrink, C. A., AND Mattart, A. V. J. De waarde van elektro- en vectorcardiografie tijdens inspanning voor de diagnose van ischemische hartziekten. Ned. Tijdschr. Geneesk. 117, 146 (1973).

4. Bartell, A. G., Behar, V. S., Peter, R. H., Orgain, E. S., and Kong, Y. Graded exercise stress test in angiographically documented coronary artery disease. Circulation 49, 348 (1974).

5. Piessens, J., Van Mieghen, W., Kesteloot, H., and de Geest, H. Diagnostic value of clinical history, exercise testing and atrial pacing in patients with chest pain. Amer. J. Cardiol. 33, 351 (1974).

6. HoRnsten, T. R. AND BRuce, R. A. Computed ST forces of Frank and bipolar exercise electrocardiograms. Amer. Heart J. 78, 346 (1969).

7. McHenry, P. L., Stowe, D. E., And Lancaster, M. C. Computer quantitation of the ST-segment response during maximal treadmill exercise: clinical correlation. Circulation 38, 691 (1968).

8. Sheffield, L. T., Holt, J. H., Lester, F. M., Conroy, D. V., and Reeves, T. J. On-line analysis of the exercise electrocardiogram. Circulation 40,935 (1969).

9. BLomovist, C. G. The Frank lead exercise electrocardiogram; quantitative study based on averaging technic and digital computer analysis. Acta Med. Scand. 178, (Suppl. 440) (1965).

10. Dower, G. E., Bruce, R. A., Pool, J., Simoons, M. L., Niederberger, M. W., ANd Meilink, L. J. Ischemic polarcardiographic changes induced by exercise; a new criterion. Circulation 38,725 (1973).

11. Rautaharju. P. M., Punsar, S., Blackburn, H., Warren, J., and Menotti, A. Waveform patterns in Frank lead rest and exercise electrocardiograms of healthy elderly men. Circulation 48, $541(1973)$.

12. Ascoop, C. A., Distelbrink, C. A., de Lang, P., and van Bemmel, J. H. Quantitative comparison of exercise vectorcardiograms and findings at selective coronary arteriography. $J$. Electrocardiol. 7,9 (1974).

13. Ascoop, C. A. "ST Forces during Exercise," Thesis, Groningen, The Netherlands, 1973.

14. Distelbrink, C. A., Ascoop, C. A., and de Lang, P. J. The diagnostic value of exercise electrocardiograms. In "Proceedings of the First European Congress on Electrocardiology," Wiesbaden. 1974.

15. Simoons, M. L. ANd Hugenholtz, P. G. Gradual changes of ECG waveform during and after exercise in normal subjects. Circulation 52, 563 (1975).

16. Simoons, M. L., Boom, H. B. K., ANd Smallenburg, E. On-line processing of orthogonal exercise electrocardiograms. Comput. Biomed. Res. 8, 105 (1975).

17. CoRnfield, J. Statistical classification methods. In "Computer diagnosis and diagnostic methods: Proceedings," (J. A. Jacquez, Ed.). p. 108. C. C. Thomas, Springfield, Ill., 1972.

18. Cornfield, J., Dunn, R. A., Batchlor, C. D., and Pipberger, H. V. Multigroup diagnosis of electrocardiograms. Comput. Biomed. Res. 6, 97 (1973).

19. Isaacs, J. H., Mills, H., and Cole, S. The post exercise T loop. In "Proceedings of the XIth International Vectorcardiography Symposium" (I. Hotiman, Ed.), p. 70. North-Holland, Amsterdam and London, 1971.

20. Cohn, P. F., Gorlin, R., Vokonas, P. S., Williams, R. A., and Herman, M. V. A quantitative clinical index for the diagnosis of symptomatic coronary-artery disease. $N$. Engl. J. Med. 286, 901 (1972).

21. Dower, G. E. "Polarcardiography", Chap. 18, p. 225. C. C. Thomas, Springfield, Ill., 1971. 